\title{
Childhood Conventional Osteosarcoma
}

National Cancer Institute

\section{Source}

National Cancer Institute. Childhood Conventional Osteosarcoma. NCI Thesaurus. Code C6590.

A high grade malignant bone-forming mesenchymal neoplasm that produces osteoid and occurs in childhood. It arises from the medullary portion of the bone. It affects the long bones, and most commonly, the distal femur, proximal tibia, and proximal humerus. Pain with or without a palpable mass is the most common clinical presentation. It usually has an aggressive growth and may metastasize through the hematogenous route. The lung is the most frequent site of metastasis. 